\title{
Role of geo-potential models in gravity field determination
}

\author{
Niraj Manandhar ${ }^{1}$ and Rene Forsberg $^{2}$ \\ 1 Chief Survey Officer, Survey Department, Geodetic Survey Branch \\ M.Eng. Geodesy(University of New South Wales,Sydney Australia) \\ manandhar_niraj@hotmail.com \\ 2. Geodynamics Department \\ Danish National Space Center, rf@space.dtu.dk
}

\section{Keywords \\ EGM (Earth gravity model), GEM (Goddard Earth Model).}

\begin{abstract}
This paper sets out to describe the developments of geopotential models and its role in gravity field determination. The paper also focuses in different geopotential models those are available and in use from 1980 onwards till at present with major emphasis placed on WGS84 EGM96 geopotential model.
\end{abstract}

\section{Introduction:}

High degree spherical harmonic expansions can provide valuable information on the earth's gravity field. Such field can be used to determine geoid undulations as well as signals to be expected from a satellite tracking mission. In the past few years the description of the earth's gravity potential in terms of spherical harmonic coefficients has been extended to degree and order $n_{\max }=360$. These high degree expansions can be used to evaluate quantities such as geoid heights, gravity anomalies, gravity disturbances and deflection of vertical with respect to geocentric ellipsoid. The theory of spherical harmonics used to determine the potential of the earth is given in Heiskanen and Moritz (1967) and Torge (1991).

The coefficients $\mathrm{C}_{\mathrm{nm}}$ and $\mathrm{S}_{\mathrm{nm}}$ up to degree (n) and order (m) for high order geopotential model are determined by a combination of data from analysis of orbit perturbation of satellites, mean terrestrial gravity values from different sized blocks and $\mathrm{N}$ values at cross over points in ocean areas measured by satellite altimetry. The long wavelength geoidal feature determined from satellite analysis are found in the low order terms of the model whilst the short wavelength, higher frequency information found from the satellite altimetry and terrestrial gravity is reflected in the higher order terms.Geopotential models to degree 180 are capable of detecting geoidal features with a half wavelength of $1^{0}$, or $110 \mathrm{~km}$ to an accuracy of $0.5 \mathrm{~m}$ in Canada and $0.2 \mathrm{~m}$ in Austrlia.

\section{Gravity Anomaly and Geopotential Coefficients}

The satellite determination of the gravity field has been long wavelength where the maximum degrees in the spherical harmonic expansion has reached to degree 360 at present. In order to obtain high frequency information, methods were developed to combine satellite-derived potential coefficient information with surface gravity data. Many new techniques had been applied to combine data to achieve more accurate and representative expansions of the Earth's gravitational potential and besides its many uses in physical geodesy it can define the reference surface for geodetic calculations involving geoid determination using the surface gravity data of the particular region or country. The geopotential models may be used for simulation studies for space missions ( Schutz et al. 1989) and the models may be used to study the spectrum of earth's gravitational field.

The spherical harmonic representation of the earth's gravitational potential $\mathrm{V}$ is defined as

$$
V(r, \theta, \lambda)=\frac{G M}{r}\left[1+\sum_{n=2}^{\infty}\left(\frac{a}{r}\right)^{n} \sum_{m=-n}^{n} c_{n m} Y_{n m}(\bar{\theta}, \lambda)\right]
$$

Where $r$ is the geocentric distance, $\theta$ geocentric colatitude, and $\lambda$ is the longitude, GM is the geocentric gravitational constant, while a (usually the equatorial radius of an adopted mean- earth ellipsoid) is the scaling factor associated with 
the fully normalized spherical geopotential coefficients, $C_{m m}$. In addition

$$
\begin{aligned}
& Y_{n m}(\theta, \lambda)=P_{n m}(\cos \theta) \operatorname{cosm} \lambda \\
& Y_{n m}(\theta, \lambda)=P_{n m}(\operatorname{Cos} \theta) \operatorname{Sinm} \lambda
\end{aligned}
$$

$P_{n m}(\operatorname{Cos} \theta)$ are the fully normalized associated Legendre functions of the first kind (Heiskanen and Moritz, 1967)

\section{The Commonly used Geopotential models}

The most commonly used higher order geopotential models and the input data used to determine them is given in Table-1.
- $\quad$ Satellite laser ranging to LAGEOS ( $\mathrm{i}=110$ degree) and satellite ( $\mathrm{i}=49.8$ degree)

- Surface gravity data which consisted of 11, 688,136 gravity values referenced to IGSN or the WGS84 ellipsoid.

- Oceanic geoid heights deduced from a set of approximately 4.2 million along track point geoid heights derived from SEASAT-1.

- NAVSTAR GPS data which consisted of simultaneous tracking data from GPS satellites.

- Lumped coefficients, "which are values of certain linear combination of gravity coefficients for rather large orbital perturbations on particular satellites.”

\begin{tabular}{|c|c|c|c|c|}
\hline Model & Degree & Origin & Date & Input Data \\
\hline OSU81 & 180 & Rapp & 1981 & GEOS 3 data $+1{ }^{0}$ Surface gravity + SEeASAT altimetry \\
\hline GRIM 3 & 36 & Reigher & 1983 & Satellite tracking data \\
\hline GEM-L2 & 20 & Lerch & 1984 & SLR+GEM9 data \\
\hline GEM2 & 200 & Wenzel & 1985 & GEM-L $2+1^{O}$ surface gravity + altimeter data \\
\hline GEM-T3S & 50 & Lerch et al. & 1992 & Satellite tracking data \\
\hline GEM-T3 & 50 & Lerch et al. & 1992 & $\begin{array}{l}\text { Satellite tracking data,altimeter data } \& \text { surface gravity } \\
\text { observation }\end{array}$ \\
\hline OSU 91A & 360 & $\begin{array}{l}\text { Rapp, Wang, } \\
\text { \& Pavlis }\end{array}$ & 1991 & Satellite tracking data(GEM-T2)+sea surface topography \\
\hline EGM 96 & 360 & $\begin{array}{l}\text { GSFC/DMA/ } \\
\text { Rapp }\end{array}$ & 1996 & Satellite tracking data + surface gravity etc. \\
\hline
\end{tabular}
( Defence Mapping Agency, 1987)

Table 1

\section{The World Geodetic System 1984 Earth ravity Model (WGS84 EGM 96)}

The WGS84 EGM is a spherical harmonic expansion of the geopotential. It consists of 32,755 geopotential coefficients and is complete through degree(n) and order(m) 180. These coefficients are determined in two separate solutions.

\section{Determination of Gravitation Coefficients}

The WGS 84 coefficients through $n=m=41$ were obtained from a weighted least square solution of a normal equation matrix developed by combining individual normal equation matrices formed from:

- Doppler tracking of seven satellite with different inclinations

After the $n=m=41$ portion of the WGS84 EGM had been solved from the weighted least square solution, the effect or contribution of the $n=m=41$ coefficients were removed from the 1 degree $\mathrm{X} 1$ degree mean free air gravity anomaly field. The WGS84 EGM coefficients from $n=42 \mathrm{~m}=0$ through $\mathrm{n}=\mathrm{m}=180$ were then determined independently via spherical harmonic analysis using residual field.

The total coefficients set $n=m=0$ through $n=m=180$ forms the WGS84 EGM, which can be used to calculate WGS 84 geoid heights, WGS $84 \Delta \mathrm{g}$ or deflection of vertical and 1 degreeX 1 degree mean gravity anomalies. 


\section{Applications of WGS84 EGM}

The EGM through $n=m=41$ is appropriate for satellite orbit calculation and prediction purposes. The use of higher order terms is not recommended. The expansions from $n=m=41$ to $n=m=180$ degree and order are needed to accurately model geoid heights $(\mathrm{N})$, and gravity disturbance components ( or deflection of vertical) and mean gravity anomalies.

The EGM validation tests rather than coefficient accuracy test were performed. This was because these comparisons would not be independent since the data being used to validate the EGM is for the most part very similar to the information used in its development. The validation test did however indicate that the data sets were integrated correctly into the EGM development process in a relative sense.

The WGS 84 is significant improvement on it existing geopotential models because of the better physical modeling, through a selection of a larger gravitational potential parameter sets secondly improved equation development, including the statistical treatment of unmodelled effects and lastly the use of newer and more accurate supporting data. Therefore EGM 96 is now globally used reference height datum.

\section{The WGS 84 Geoid}

The geoid is defined as that particular equipotential surface of the earth that coincides with the mean sea level over the oceans and extend hypothetically beneath all land surfaces. In a mathematical sense, the geoid is also defined as so many meters $(+\mathrm{N})$ or below $(-\mathrm{N})$ the ellipsoid.

The WGS 84 geoid can be defined by superimposing "N" values, determined by a spherical harmonic expansion, on the WGS 84 ellipsoid. WGS 84 geoid heights (N) are calculated using a spherical harmonic expansion and the WGS EGM through $n=m=180$. These geoid heights when combined with WGS 84 ellipsoid define the WGS 84 geoid.

The principle function of the WGS 84 geoid (Decker, 1986) are to serve as a reference surface for WGS 84 related heights above mean sea level (h) values in those parts of the world where geodetic vertical datum are not available from tide guage data. (Enabling $\mathrm{H}=\mathrm{h}$ (WGS 84) $-\mathrm{N}$ (WGS 84). Secondly its principle function is to provide WGS 84 positional data in oceanic regions where WGS 84 geoid heights are equivalent to WGS 84 geodetic heights that is $\mathrm{H}$ (WGS 84) $=\mathrm{h}$ (WGS 84) and lastly to provide the measure of how accurately the WGS 84 ellipsoid approximates the mean sea level surface.
The RMS of a WGS 84 geoid height taken on the basis of world wide 1 degree $\mathrm{X} 1$ degree grid, is 30.5 meter. This RMS value is an indication of how accurately the WGS 84 ellipsoid taken as a mathematical figure of the earth, fits the earth's mean sea level surface. WGS 84 geoid heights determined using spherical harmonic expansion based on the WGS 84 EGM are accurate to $+/-2$ to 3 meters over approximately $70 \%$ of the earth, while $93 \%$ of the earth has WGS 84 geoid heights that are accurate to better than +/- 4 meters.

For the application in geophysics, lower order surveys and the mapping control, N (WGS 84) may well be sufficient. However, for geodetic and oceanographic purposes, higher resolution geoids will be required to improve relative accuracy to the order of 1 to $2 \mathrm{ppm}$.

\section{GEM-T3 and GEM-T3S Models}

The improved models of the earth's gravitational field have been developed from conventional satellite tracking data (GEM-T3S) and from the combination of satellite tracking, satellite altimeter and surface gravity data (GEM-T3). This combination model represents a significant improvement in the modeling of the gravity field at half wavelength of 350 $\mathrm{km}$ and longer. Both models are complete to degree and order 50. The GEM-T3 model provides for more accurate computation of satellite orbital effect as well as giving a superior geoidal representation from that achieved in any previous Goddard Earth Model (GEM). A description of the model, their development and an assessment of their accuracy has been given by Lerch et al.(1992).

\section{The Ohio State University (OSU) Series}

The series of models of high order models for use in geoid studies and were developed by Professor Richard Rapp and other fellow researchers. The degree of the model has increased with time, and bases of the models has similarly become more sophisticated. The OSU models had been used widely throughout the world as a reference model for precise local geoid determination.

The OSU 91A model is again the result of the work carried out by Rapp et al.(1991) and it is computed to degree 360 . Besides incorporating new anomaly and altimeter data, it also includes a sea surface topography model to degree $10 / 15$, and adjusted GEOSAT orbits for the first year of the exact repeat mission (ERM). It was started from the GEMT2 potential coefficient model and its error covariance matrix and GEOSAT orbits computed by Haines et al. (1990) using GEM - T2 Model. This model has also been tested through orbit predictions and data fitting, through 
comparisons with geoid undulations computed from Doppler and GPS located stations, and with comparisons to geoid undulations implied by GEOSAT altimeter data.

\section{Conclusion}

Geopotential models definitely play a very important role in modeling the gravity field of the earth. Without the use of the geopotential models it would be very difficult to accurately support terrestrial extraterrestrial endeavors. In this paper various model have been mentioned with major emphasis on WGS 84 EGM, GEM-T series and OSU series. Apart from that for a long period the OSU91A remained as a most accurate reference model for many application in geodesy and geosciences but it is now replaced by EGM96 geopotential model. The growing accuracy of the satellite borne data, satellite altimeter data, airborne gravity data, surface gravity data definitely calls for more accurate geopotential models.

\section{Referances}

1. Decker, B.L (1986). World Geodetic System 1984, Fourth International Geodetic Symposium on Satellite Positioning (Austin, Texas, 28 April -2 May 1986)

2. Denker, H. and Rapp,R. (1990). Geodetic and Oceanographic Results from the Analysis of 1 year of Geosat Data, J. Geoshys. Res.,95,C8 pp.1315113168, 1990.

3. Groten, I.E. (1979). Geodesy and the Earth's Gravity Field (Vol. 1: Principles and Conventional Methods). Fred Dummler Verlag: Bonn, 1979.

4. Heiskanen, W. and Moritz, H. (1967) Physical Geodesy, Freeman: San Francisco, 1967.
5. Kearsley, A.H.W.(1988), The determination of geoid-ellipsoid separation for GPS leveling. The Australian Surveyor Vol.34 No.1 March1988.

6. Lerch, F.J.,R.S. Nerem, B.H. Putney, T.L. Felsentreger, B.V.Sanchez, S.M. Klosko,G.B. Patel, R.G. Williamson, D.S. Chinn, J.C. Chan, K.E. Rachlin, N.L. Chandler, J.J. McCarthy, J.A. Marshall, S.B. Luthcke, D.W. Palvis, J.W. Rpbbins, S. Kapoor and E.C. Palvis (1992), Geopotential Models of the Earth From Satellite Tracking, Altimeter and Surface Gravity Observations: GEM - T3 and GEM -T3S, NASA Technical Memorandum 104555, Goddard Space Flight Center: Greenbelt, MD. January 1992.

7. Rapp, R.Hand Pavlis, N.K. (1990). The development and analysis of geopotential models to spherical harmonic degree 360. Journal of Geophysical Research, Vol. 95 No. B13, pp. 21885-21911, Dec. 10, 1990.

8. Rap, R.H., Wang, Y.M. and Pavlis, N.K. (1991). The Ohio State 1991Geopotential and Sea Surface Topography Harmonic Coefficient Models Report No. 410, Department of Geodetic Science and Surveying. The Ohio State University: Colombus, Ohio. August 1991.

9. The Defense Mapping Agency (1987). DMA Technical Report (Supplement to Department of Defence World Geodetic System 1984 Technical Report) Part 1: Methods, Techniques, and Data Used in WGS84 Development, DMA TR, 8350. 2 -A, Washington DC. 1 Dec. 1987.

10. Torge, W. (1991) Geodesy. $2^{\text {nd }}$ Edition. Walter de Gruyter: Berlin , 1991. 